\title{
Water Sorption-Desorption Test and Moisture Accumulation Test for Functional Assessment of Atopic Skin in Children
}

\author{
GIOVANNI PELLACANI and STEFANIA SEIDENARI \\ Department of Dermatology, University of Modena and Reggio Emilia, Italy
}

\begin{abstract}
Sorption-desorption and moisture accumulation tests are simple and quick methods for the in vivo functional analysis of stratum corneum hydration kinetics. The aim of this study was to evaluate the hydration dynamics of the uninvolved and affected skin of children with atopic dermatitis and to compare them with the skin of healthy children. The study investigated 45 children. The dynamic tests were performed using the corneometer CM820. Numerical parameters were calculated. With the sorption-desorption test, eczematous skin showed lower water accumulation during the sorption phase, whereas water was released more slowly during the desorption phase. With the moisture accumulation test, increases in water accumulation velocity and in water accumulation were observed in atopic children. Dynamic tests showed that the stratum corneum of unaffected atopic skin was less hydrated but more easily hydratable than normal skin. Conversely, despite a lower absorption capability, eczematous skin showed a greater avidity to retain water. New functional parameters (water-sorption capacity and accumulated water decay) are proposed to describe more precisely the hydration kinetics of eczematous skin. Key words: accumulated water decay; water-sorption capacity; water-holding capacity; skin hydration; capacitance.
\end{abstract}

(Accepted February 7, 2001.)

Acta Derm Venereol 2001; 81: 100-103

Stefania Seidenari, Department of Dermatology, University of Modena and Reggio Emilia, 41100 Modena, Italy. E-mail: seidenar@unimo.it

The stratum corneum (SC) is composed of protein-enriched corneocytes embedded in a continuous lipid-enriched intercellular matrix $(1,2)$. This compact structure not only provides defence against various environmental damage, but also protects against water loss. SC proteins, lipids and low molecular weight substances (natural moisturizing factors) bind and retain water in the $\mathrm{SC}$, maintaining the skin barrier properties.

The introduction of instrumental devices for evaluating hydration and transepidermal water loss (TEWL) has enabled objective and quantitative non-invasive evaluation of water content (3) and barrier efficiency (4) of the SC. The single measurement of hydration parameters is important in the evaluation of a steady state of the SC, but does not give information on its dynamic functions, which can only be evaluated using repeated measurements such as the water sorption-desorption test (5) and the moisture accumulation test (6).

The water sorption-desorption test is a simple and quick method for in vivo functional analysis of SC hydrationdehydration kinetics. This method provides information not only on the ability of the SC to take up water (hygroscopicity), but also on its ability to retain water, opposing a dehydration process (water-holding capacity) (5). The water sorptiondesorption test, tested on scaly skin conditions, such as psoriasis $(5,7,8)$, eczematous dermatitis $(5,9)$ and other kinds of scaly dermatoses $(5,10)$, showed evidence of a decreased capability of affected skin to bind water compared with normal skin. The water sorption-desorption test has also been applied to assess the effects of different moisturizers $(6,11-14)$ under different experimental conditions of impairment of the skin barrier function $(6,14-18)$.

The moisture accumulation test, which evaluates the accumulation of unbound water occurring when occlusive contact is maintained over a short period, was developed to determine endogenous unbound water diffusion across the SC in vivo (6) and subsequently used to assess the SC after treatment with irritants $(6,14)$ and moisturizers (14).

Skin dryness is a typical feature of atopic dermatitis (AD) and it seems to be related to abnormalities of the horny layer, especially of the lipid components (19). The skin of patients with AD has been non-invasively described using instruments which evaluate the water content $(9,20-23)$ and the evaporation rate through the epidermis (22-25). However, no data have been reported so far on the dynamic properties of the uninvolved and eczematous skin of children affected by AD.

The aim of this study was to evaluate the SC hydration kinetics in uninvolved and affected skin of children with AD using water sorption-desorption and moisture accumulation tests and to compare them with the skin of healthy children.

\section{MATERIAL AND METHODS}

Study subjects

Forty-five children, 21 girls and 24 boys, aged $4-12$ years (mean \pm SD, $8 \pm 3$ years), comprising 15 healthy subjects, 15 patients with AD disease who had been disease free for at least one month, and 15 patients with AD with visible eczematous lesions, were studied. Atopic patients included in the study were selected according to Hanifin \& Rajka (26). The 3 groups were homogeneous in sex and age distribution.

\section{Instrument}

The corneometer CM 820 (Courage \& Khazaka, Germany), which measures the electrical capacitance of the skin surface, expressed in arbitrary units (a.u.), was used to assess the hydration values. Values are displayed digitally. The probe consists of a $49 \mathrm{~mm}^{2}$ glass surface, applied on the skin under a standard load of $3.65 \mathrm{~N}$, kept constant by a spring.

Test procedure

Sorption-desorption test. The water sorption-desorption test was carried out as described by Tagami et al. (5) with slight modifications, 
such as a longer water application time ( $1 \mathrm{~min}$ instead of $10 \mathrm{~s})$. An area on the volar aspect of the left forearm, $3 \mathrm{~cm}$ distant from the elbow crease, was identified. Eczematous, non-scaly, forearm skin was tested on affected AD subjects. The first measurement, taken before treatment, represented the prehydration state value (baseline measurement $)$. Distilled water $(50 \mu \mathrm{l})$ was then pipetted onto the skin, left for $1 \mathrm{~min}$ and wiped with a sterile gauze, and the hydration immediately measured $(60 \mathrm{~s})$. The capacitance was subsequently measured every $30 \mathrm{~s}$ for up to $2 \mathrm{~min}$.

As a traditional parameter, the water-holding capacity (5), defined as area under the curve plotted by capacitance measurements, was calculated considering the prehydration value to be equal to 0 . The difference between the capacitance measured immediately after water removal (60s) and the prehydration state (baseline) was defined as the water-sorption capacity. Moreover, $60 \mathrm{~s}$ values were considered to be equal to $100 \%$ and values referring to other measurement times were calculated accordingly. A curve was then constructed enabling the definition of a new parameter, the accumulated water decay, corresponding to the percentage of water released from the SC throughout the desorption phase (Fig. 1).

Moisture accumulation test. The moisture accumulation test was performed as described by Van Neste (6) with slight modifications (measurements were taken every $30 \mathrm{~s}$ for up to $5 \mathrm{~min}$ instead of every $3 \mathrm{~s}$ for up to $45 \mathrm{~s}$ ), on a symmetrical area on the right forearm. After the acquisition of the prehydration value (baseline measurement), the probe remained on the skin for 5 min to create occlusive conditions. The hydration value was read every 30 s without removing the probe from the skin.

Both raw and differential corneometer values were considered. The latter were obtained by subtracting the baseline value from the raw corneometer values of each subject, to exclude variations related to differences in basal skin hydration.

Water accumulation was represented by the area under the curve between baseline and $300 \mathrm{~s}$, considering the baseline value to be equal to 0 . The water accumulation velocity during the first $30 \mathrm{~s}$ was calculated from the expression: (30s - baseline)/30" (6).

\section{Statistics}

Means and standard deviations were calculated. The Mann-Whitney test for independent samples, as implemented in the SPSS statistical package (release 7.0, 1995; SPSS, Chicago, IL, USA), was used to evaluate the differences between raw and differential corneometer measurements, water-holding capacity, water-sorption capacity, accumulated water decay, water accumulation velocity and water accumulation values referring to different groups (healthy, unaffected subjects with $\mathrm{AD}$ and affected subjects with $\mathrm{AD})$. A $p$-value $<0.05$ was considered statistically significant.

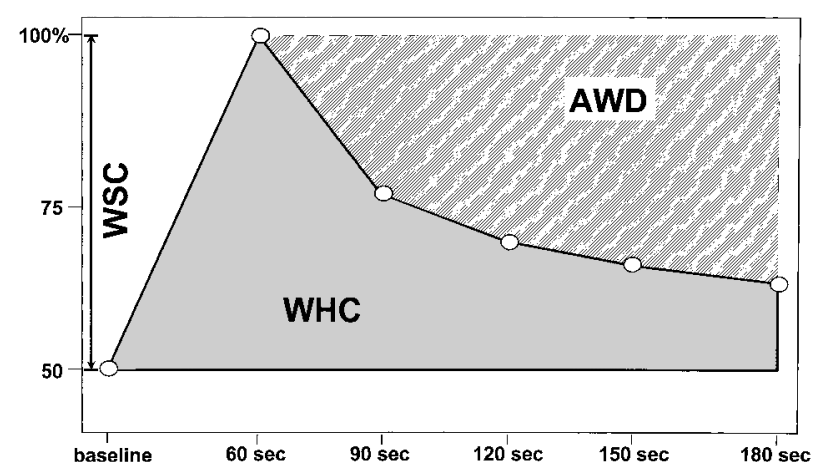

Fig. 1. Example of the sorption-desorption test curve. Traditional and new calculated parameters are highlighted. WSC: water-sorption capacity; AWD: accumulated water decay; WHC: water-holding capacity.

\section{RESULTS}

Water sorption-desorption test

Baseline values were: healthy subjects $=49.9 \pm 6.8$, unaffected skin of patients with $\mathrm{AD}=41.2 \pm 8.3$, and affected skin of patients with $\mathrm{AD}=31.4 \pm 9.0$.

Significant differences between healthy and unaffected subjects with $\mathrm{AD}$ were observed only for baseline values, whereas affected patients with AD had significantly lower values for each measurement.

Water-holding capacity values were apparently higher in healthy atopic skin and lower in eczematous skin, although the differences were not statistically significant.

During the sorption phase (Fig. 2), the amounts of water accumulated in healthy and unaffected AD skin were equal, whereas eczematous skin had a lower water-sorption capacity. During the desorption phase (Fig. 2), water was released more rapidly by healthy skin than by atopic skin. Moreover, the percentage release of accumulated water was significantly lower at eczematous skin sites.

\section{Moisture accumulation test}

Baseline values were: healthy subjects $=47.9 \pm 7.3$, unaffected skin of patients with $\mathrm{AD}=42.7 \pm 5.8$, and affected skin of patients with $\mathrm{AD}=31.8 \pm 9.5$.

Significant differences were observed for baseline values in the 3 groups. Lesional skin had higher water accumulation values compared with the other groups.

Differential corneometer values are plotted in Fig. 3. Each differential corneometer value and water accumulation velocity were significantly higher in AD lesional skin in respect of both healthy and unaffected AD skin. Measurement at $30 \mathrm{~s}$ and water accumulation velocity had significantly increased in unaffected subjects with AD compared with healthy subjects. From $30 \mathrm{~s}$ up to $300 \mathrm{~s}$ occlusion the differential corneometer values increased progressively at a steady rate in both atopic unaffected skin and healthy subjects.

\section{DISCUSSION}

TEWL and capacitance measurements are widely used to evaluate the properties of the SC and its variations both in physiological and pathological conditions. The skin of atopic subjects has been thoroughly studied, showing that not only affected skin, but also apparently healthy unaffected skin presents lower capacitance values and higher TEWL values (23). These data have been correlated with the reduced amount of lipids observed in atopic skin, suggesting that a decrease in lipids, in particular of ceramides, in the SC is involved in barrier impairment in AD skin (19).

Imokawa \& Hattori (27) showed that the chemical extraction of skin-surface lipids induced a decrease in both skin hydration and water-holding capacity, suggesting that the structural lipids may play a considerable role in the waterretention properties of the SC.

Simple and quick tests such as water sorption-desorption test and moisture accumulation test allow the dynamic description of skin functions. Whereas the water sorption-desorption test evaluates the capability of the SC to retain water from the environment, the moisture accumulation test focuses on the kinetics of endogenous water diffusing across the SC. 


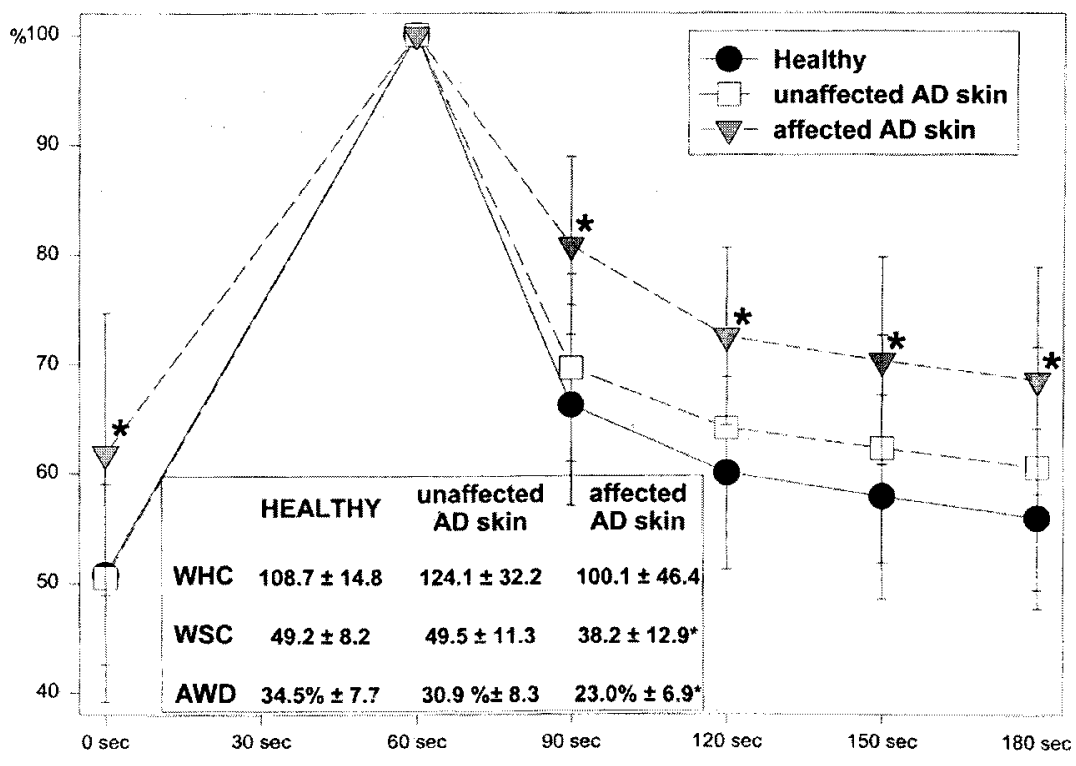

Fig. 2. Sorption-desorption test performed on healthy skin, unaffected atopic dermatitis skin and eczematous skin. The curves were plotted with the $60 \mathrm{~s}$ value equal to $100 \%$ and values referring to other measurement times were calculated accordingly. WSC: water-sorption capacity; AWD: accumulated water decay; WHC: water-holding capacity. *Statistically significant differences $(p<0.05)$ compared with healthy skin.

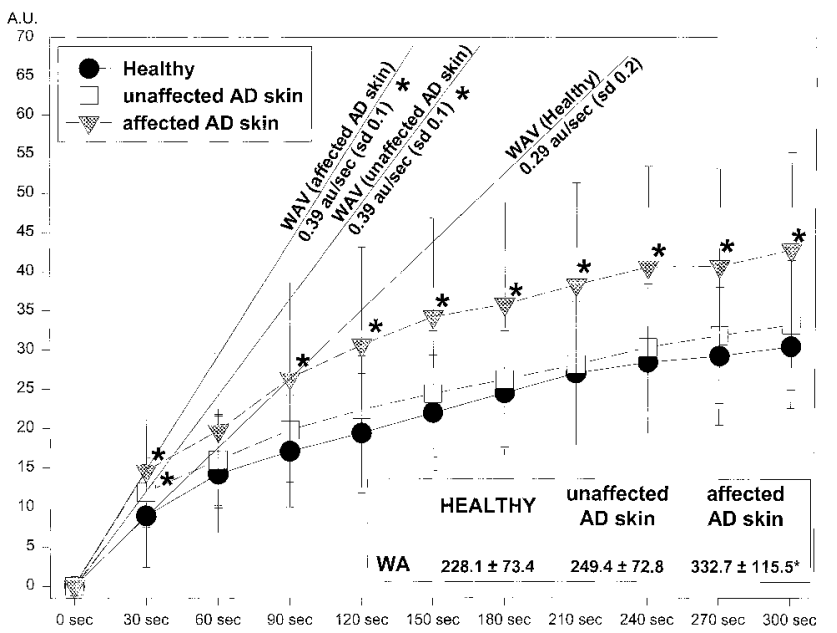

Fig. 3. Moisture accumulation test. Differential corneometer values as measured on healthy skin, unaffected atopic dermatitis skin and eczematou s skin. WA: water accumulation; WAV: water accumulation velocity; A.U.: arbitrary units. *Statistically significant differences $(p<0.05)$ compared with healthy skin.

In scaly skin conditions a good correlation between the scaling process and the decreased water-holding capacity was shown, and clinical improvement in the pathological condition after treatment was linked to increased water retention (5). Moreover, the water-retention effect, measured by the increase in the prehydration state, water-holding capacity, water accumulation and water accumulation velocity, after application of urea-containing products on normal skin, was also observed, indicating that hydrophilic molecules hold water coming both from the surface and from the deeper epidermis (14). However, the same effects on water-holding capacity, water accumulation and water accumulation velocity were observed by the application of irritants, such as sodium lauryl sulphate. In the latter case, the increase in water-holding capacity was attributed to the impairment of the cutaneous barrier, which allowed the water to penetrate more easily into the SC (14), and the increase in water accumulation and water accumulation velocity was correlated with the parallel increase in TEWL $(28,29)$. Therefore, the dynamics of water accumulation and release in different spontaneous and experimental skin conditions is not sufficiently described by water-holding capacity (the "traditional" parameter which represents a "global" measurement of water uptake and release) and does not take into account the different phases of the test characterizing different skin situations.

In the present study, baseline capacitance values were significantly lower in subjects with AD than in healthy subjects, confirming previous data $(19,23)$. The water-holding capacity, as calculated according to Fig. 1, was lower for eczematous skin, which behaves like healthy skin subjected to delipidization by organic solvents (27).

After the application of water, the relative increase in capacitance values was similar in healthy subjects and in patients with $\mathrm{AD}$ at unaffected skin sites. In contrast, the amount of water absorbed by eczematous skin was lower. The second part of the sorption-desorption curve shows that the behaviour of unaffected AD skin and that of healthy subjects is the same, with the former showing only slightly higher, but non-significant, differential corneometer values compared with the latter. Conversely, a greater avidity of eczematous skin is indicated by a lower accumulated water decay. Therefore, surprisingly, the lower water-holding capacity of eczematous skin does not seem to be due to a reduced ability to retain water (in fact, less water is released, as demonstrated by the descending part of the graph in Fig. 2), but is probably due to a decreased absorption capability, as shown by the ascending part of the graph.

With the moisture accumulation test, increases in water accumulation velocity and water accumulation were observable in children with AD. This could be related to increased water flux from the deep epidermis through the SC, due to the impairment of skin barrier. When the moisture accumulation test was performed on eczematous skin, an increase in water accumulation velocity and water accumulation values was 
noticed compared with both unaffected and healthy skin. In accordance with these results, Van Neste demonstrated a direct correlation between water accumulation velocity and water accumulation values and TEWL values in subjects with $\mathrm{AD}$, by performing the moisture accumulation test with different concentrations of sodium lauryl sulphate (6).

In conclusion, whereas the single measurement of hydration is important in order to define a steady state of the SC, the water sorption-desorption test and moisture accumulation test enable its dynamic properties to be assessed. Dynamic tests on unaffected children with AD showed that the SC is less hydrated, but more easily hydratable with water coming either from the skin surface (water sorption-desorption test) or from the deeper epidermis (moisture accumulation test). Moreover, the hydration dynamics of the skin of unaffected subjects with AD can be compared with the results of Treffel \& Gabard (14), who investigated subclinical irritation induced by sodium lauryl sulphate at a low concentration. Specific alterations in the SC of patients with AD could therefore lead to a subclinical state of irritation, which renders environmental factors more effective in inducing damage in atopic than in normal skin, thus causing the phenotypic expression of eczema.

\section{REFERENCES}

1. Elias PM. Epidermal lipids, barrier function and desquamation. J Invest Dermatol 1983; 80: 44s-49s.

2. Yamamoto A, Serizawa S, Ho M, Sato Y. Stratum corneum lipid abnormalities in atopic dermatitis. Arch Dermatol Res 1991; 283: $219-223$.

3. Tagami H, Ohi M, Iwatsuki K, Kanamaru Y, Yamada M, Ichijo B. Evaluation of skin surface hydration in vivo by electrical measurement. J Invest Dermatol 1980; 75: 500-507.

4. Baker H, Kligman AM. Measurement of transepidermal water loss by electrical hygrometry. Instrumentation and responses to physical and chemical insults. Arch Dermatol 1967; 96: 441-452.

5. Tagami H, Kanamaru Y, Inoue K, Suehisa S, Inoue F, Iwatsuki $\mathrm{K}$, et al. Water sorption-desorption test of the skin in vivo for functional assessment of the stratum corneum. J Invest Dermatol 1982; 78: 425-428.

6. Van Neste D. In vivo evaluation of unbound water accumulation in stratum corneum. Dermatologica 1990; 181: 197-201

7. Borroni G, Vignati G, Brazzelli V, Vignoli GP, Gabba P, Gatti $\mathrm{M}$, et al. Changes in the water holding capacity of psoriatic stratum corneum in vivo. Acta Derm Venereol 1989; Suppl 146: $192-194$.

8. Tagami H. Quantitative measurements of water concentration of the stratum corneum in vivo by high-frequency current. Acta Derm Venereol 1994; Suppl 185: 29-33.

9. Thune P. Evaluation of the hydration and the water-holding capacity in atopic skin and so called dry skin. Acta Derm Venereol 1989; Suppl 144: 133-135.

10. Urano-Sueisha S, Tagami H. Functional and morphological analysis of the horny layer of pityriasis alba. Acta Derm Venereol 1985; 65: 164-167.

11. Stender IM, Blichmann C, Serup J. Effects of oil and water baths on the hydration state of the epidermis. Clin Exp Dermatol 1990; 15: 206-209.

12. Sindhvananda J, Gritiyarangsan $P$, Rungrairatanaroij $P$, Kullavanijaya P. Hygroscopicity and water-holding capacity of moisturizing agents: a single-application in vivo study. J Soc Cosmet Chem 1993; 44: 279-288.

13. Martinsen OG, Grimnes S, Karlsen J. Electrical methods for skin moisture assessment. Skin Pharmacol 1995; 8: 237-245.

14. Treffel P, Gabard B. Stratum corneum dynamic function measurements after moisturizer or irritant application. Arch Dermatol Res 1995; 287: 474-479.

15. Miyauchi $\mathrm{H}, \mathrm{H}$ orio $\mathrm{T}$, Asada $\mathrm{Y}$. The effect of ultraviolet radiation on the water-reservoir functions of the stratum corneum. Photodermatol Photoimmunol Photomed 1993; 9: 193-197.

16. Okah FA, Wickett R, Pompa K, Hoath SB. Human newborn skin: the effect of isopropanol on skin surface hydrophobicity. Pediatr Res 1994; 35: 443-446.

17. Graves CJ, Edward C, Marks R. The effects of protective occlusive gloves on stratum corneum barrier properties. Contact Dermatitis 1995; 33: 183-187.

18. Brazzelli V, Distante F, Peroni G, Berardesca E. Effects of systemic treatment with statins on skin barrier function and stratum corneum water-holding capacity. Dermatology 1996; 192: 214-216.

19. Di Nardo A, Wertz P, Giannetti A, Seidenari S. Ceramide and cholesterol composition of the skin of patients with atopic dermatitis. Acta Derm Venereol 1998; 78: 27-30.

20. Werner Y. The water content of the stratum corneum in patients with atopic dermatitis. Measurement with the corneometer CM 420. Acta Derm Venereol 1986; 66: 281-284.

21. Lodén M, Olsson H, Axéll T, Werner Linde Y. Friction, capacitance and transepidermal water loss (TEWL) in dry atopic skin and normal skin. Br J Dermatol 1992; 126: 137-141.

22. Berardesca E, Fideli D, Borroni G, Rabbiosi G, Maibach H. In vivo hydration and water-retention capacity of stratum corneum in clinically uninvolved skin in atopic dermatitis and psoriatic patients. Acta Derm Venereol 1990; 70: 400-404.

23. Seidenari S, Giusti G. Objective assessment of the skin in children affected by atopic dermatitis: a study of $\mathrm{pH}$, capacitance and TEWL in eczematous and clinically uninvolved skin. Acta Derm Venereol 1995; 75: 429-433.

24. Abe T, Ohkido M, Yamamoto K. Studies on skin surface barrier functions, skin surface lipids and transepidermal water loss in atopic skin during childhood. J Dermatol 1978; 5: 223-229.

25. Werner $Y$, Lindberg M. Transepidermal water loss in dry and clinically normal skin in patients with atopic dermatitis. Acta Derm Venereol 1985; 65: 102-105.

26. Hanifin JM, Rajka G. Diagnostic features of atopic dermatitis. Acta Derm Venereol 1980; Suppl 92: 44-47.

27. Imokawa G, Hattori M. A possible function of structural lipids in the water-holding properties of the stratum corneum. J Invest Dermatol 1985; 84: 282-284.

28. Agner T, Serup J. Sodium lauryl sulphate for irritant patch testing - a dose-response study using bioengineering methods for determination of skin irritation. J Invest Dermatol 1990; 95: 543-547.

29. Wilhelm KP, Cua AB, Wolff HH, Maibach HI. Surfactantinduced stratum corneum hydration in vivo: prediction of the irritation potential of anionic surfactants. J Invest Dermatol 1993; 101: $310-315$. 